\title{
ANTERIOR DISLOCATION OF THE ULNA AT THE INFERIOR RADIO-ULNAR JOINT
}

\author{
Case Reports, with a Discussion of the Anatomy of Rotation of the Forearm
}

\author{
A. P. Rose-Innes, Cape Town, South Africa
}

From the Groote Schuur Hospital, Cape Town

A highly characteristic but nevertheless rare injury due to an apparently simple and commonplace pattern of violence is always interesting. Simple anterior dislocation of the distal end of the ulna was first described in 1777 by Desault in a cadaver (Cotton and Brickley 1912); by 1937 Vergoz and Choussat were able to collect sixty-eight cases from the literature (Cox 1942), and since then occasional cases have been reported (Hill 1939, Regan and Bickel 1945, Curr and Coe 1946, Liebolt 1950, Goulden 1954). Two cases, one of acute and one of habitual subluxation, are presented here; and an attempt is made to revise the description of movement occurring at the inferior radio-ulnar joint in rotation of the forearm, and that of the normal function of the triangular fibrocartilage.

Anterior, posterior and longitudinal dislocations of this joint occur often as components of complex deformities of the forearm. A large group complicates fractures of the distal radius and ulna or their shafts. All deformities of the elbow joint, including excessive resection of the radial head, may have an effect on the distal joint. Many disturbances of bone growth and destruction of bone may distort the joint, such as congenital posterior dislocation of the ulnar head, epiphysial injury, exostosis, diaphysial aclasis, neoplasm, osteitis, chronic arthritis, and rickets.

The acute traumatic cases form a distinct group, and habitual anterior and more commonly posterior dislocations comprise a second. The established posterior dislocations from any cause are usually loosely classed as Madelung's deformity.

The variety of conditions affecting it shows that the joint is susceptible to a wide range of abnormal mechanical stresses, and increases interest in the rarity of the simple traumatic dislocation.

\section{SIMPLE ANTERIOR DISLOCATION}

Clinical features-Occasionally dislocation is caused by direct violence-rarely a direct blow on the ulnar border of the distal forearm (Cotton and Brickley 1912); more often it is produced by the forced wrenching of a passive distal forearm into extreme supination, as in a limb caught in rotating machinery (Darrach 1912).

The usual indirect mechanism, however, seems almost specific, and can be analysed in every detailed history as forced extreme supination on a fixed wrist. Examples are: falls on to the supinated hand, wrenches sustained in cranking engines (Darrach 1912), supinating twists sustained in vaulting (Goulden 1954), weight lifting with the supinated hand (Hill 1939), and-in one instance described by Cotton and Brickley (1912)-a rotational strain sustained in wringing washing.

The presentation in the acute case is characteristic (Case 1). The forearm is painfully locked in full supination; the elbow moves freely and the radio-carpal joint and hand, less rotated than the forearm, show good mobility. There is a marked furrow on the medial side of the distal extensor aspect of the forearm, and a slight prominence on the anterior aspect of the wrist. Lateral displacement of the ulnar head gives the distal forearm a narrowed appearance, and the flexor tendons overlying the head add to the anterior prominence. A 
lateral radiograph shows the displacement clearly, and commonly the ulnar styloid process is broken at its base (Fig. 1).

Habitual simple subluxation is associated with a previous characteristic injury, and occurs whenever a similar but slighter stress is imposed on the forearm (Davidson and Horwitz 1938, Hill 1939, Regan and Bickel 1945, Liebolt 1950), or merely on unforced supination as in the case reported here (Case 2). The deformity is less because the ulnar head does not slip in front of the distal end of the radius. When reduced, in pronation, antero-posterior laxity of the inferior radio-ulnar joint can be demonstrated by manipulation. The subluxation occurs with a click, and may be disablingly painful, with weakened supination (Davidson and Horwitz 1938, Hill 1939), or quite asymptomatic (Case 2).

Treatment-The acute dislocation is reduced easily by direct pressure over the ulnar head while the forearm is pronated, and reduction is stable in this position. Severe pain is produced by this manipulation, and general anaesthesia is usually necessary. It was perhaps because of this pain that Dupuytren used an elaborate technique of manipulation requiring two assistants and countertraction from a wall. The method he used in two cases is described by Gibson (1925). Pain subsides immediately after reduction. Normal function has been obtained in several cases after reduction by manipulation and immobilisation in pronation in plaster for four to six weeks (Gibson 1925, Curr and Coe 1946, Goulden 1954).

Often the diagnosis is only made some weeks after injury. In Darrach's (1912) first case open reduction was attempted eight weeks after injury and ankylosis of the radio-ulnar joints resulted. In his second case he excised the distal inch of the ulna and succeeded in restoring full rotation.

For habitual dislocation most authors have considered excision of the distal ulna too drastic; osteotomy of the distal radius to tauten the inferior radio-ulnar ligaments has been tried and found to be ineffective (Milch 1926). Attempts to reinforce the ligaments have been favoured. An ingenious procedure of rerouting the tendon of extensor carpi ulnaris through a tunnel in the distal ulna was successful in one case (Hill 1939). Fascial slings variously encircling or passing through the distal ulna and attached to the radius have been used (Milch 1926, Davidson and Horwitz 1938, Regan and Bickel 1945, Liebolt 1950). Although several excellent results have been reported after such procedures, they do not invariably prevent antero-posterior instability, and may limit rotation (Liebolt 1950).

\section{CASE REPORTS}

Case 1-A woman aged twenty-four years (Fig. 1) attempted to break a fall on stairs and came down heavily on her right hand, twisting the forearm into forced supination on the fixed hand. When examined two hours later the forearm was found locked in supination; the wrist showed a deep depression posteriorly at the site of the ulnar head and a slight mid-anterior prominence deep to the flexor tendons. There was severe pain, much increased by any attempt to rotate the forearm. There was full movement of the radio-carpal joint and fingers. The patient had paraesthesia over the palmar aspect of the hand and fingers, most marked in the thumb and index finger, but there was no objective motor or sensory loss in the hand. A radiograph showed anterior dislocation of the ulnar head with a fracture of the ulnar styloid process. Under general anaesthesia the dislocation was reduced with a distinct click by direct pressure on the anterior prominence combined with pronation. The wrist was immobilised in an above-elbow plaster with the forearm in mid-rotation. Reduction was confirmed by a further radiograph. Immobilisation was continued for six weeks. A week after removal of the plaster full rotation of the forearm had been recovered, and there was no instability of the inferior radio-ulnar joint.

Case 2-A woman aged twenty-six years (Figs. 2 to 4 ) injured her left wrist ten years ago during gymnastics, twisting the arm into supination while gripping a bar. The forearm was manipulated 


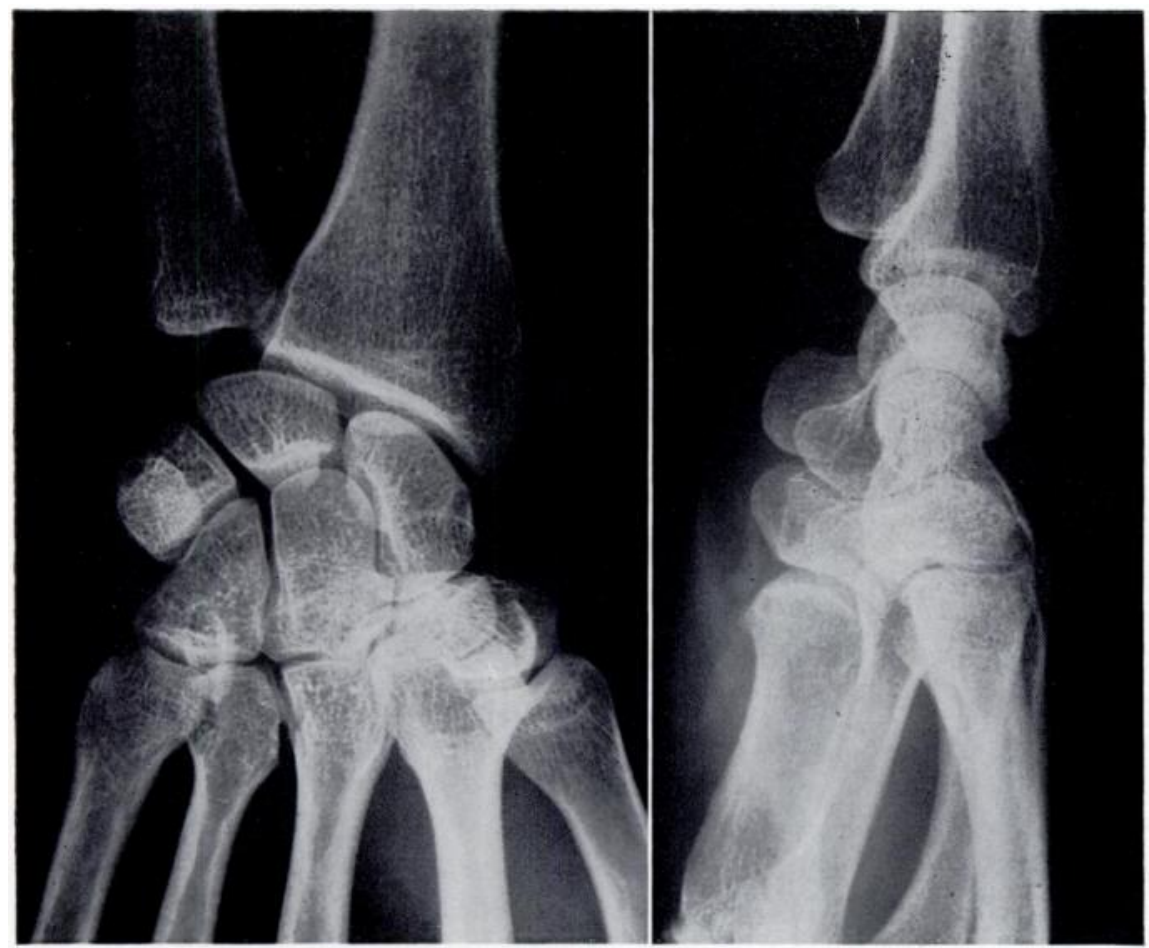

Fig. 1

Case 1-Radiographs of the wrist.

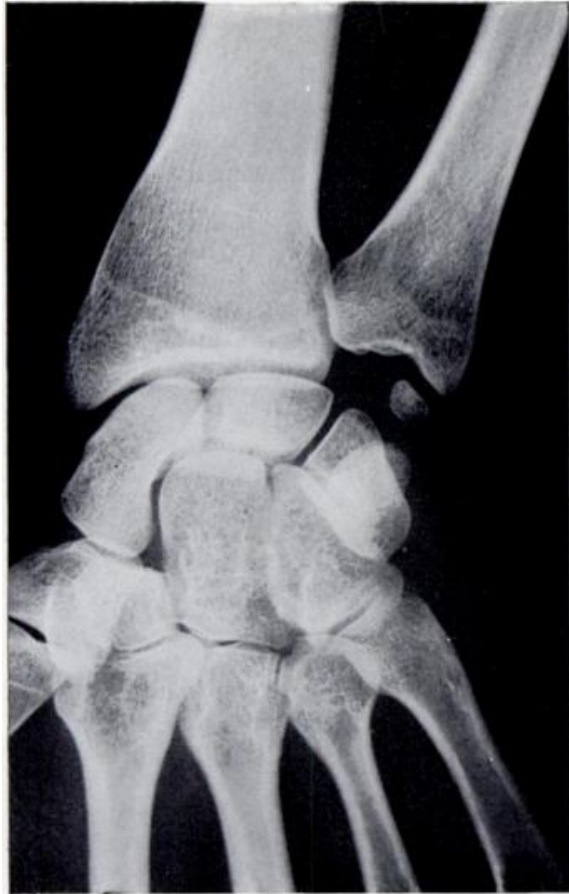

FIG. 2

Case 2-Antero-posterior radiograph.

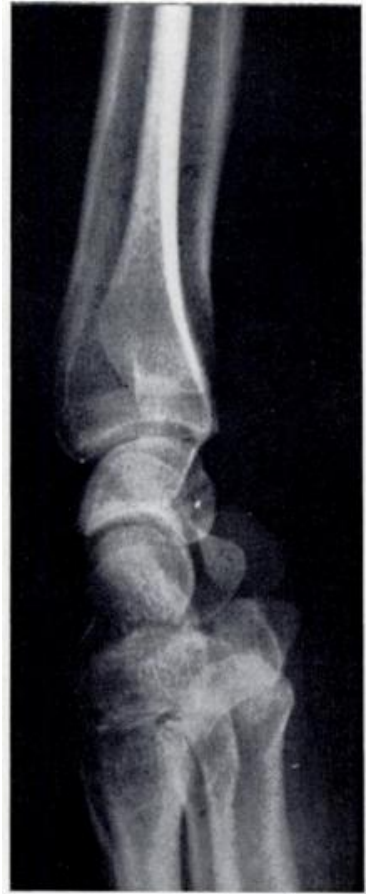

Fig. 3

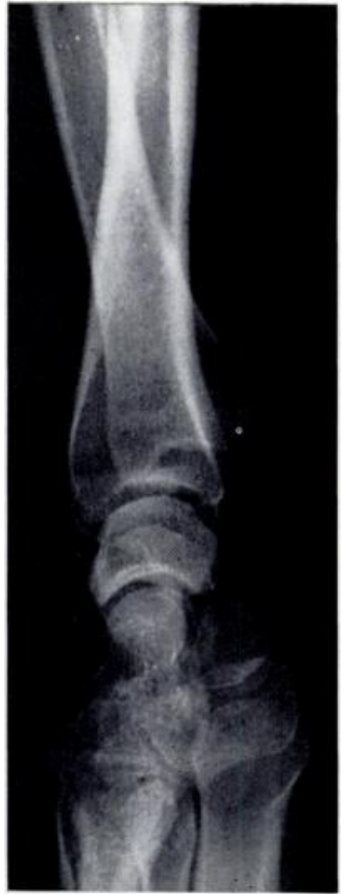

FIG. 4

Case 2-Lateral radiograph in pronation (Fig. 3) and in supination (Fig. 4), showing subluxation of the ulna.

vol. 42 B, No. 3, AUgust 1960 
by a doctor soon afterwards and she was allowed to use the limb without restraint. The wrist became comfortable in about two weeks. During the subsequent year the wrist was unstable, painful and weak when stress was put on it in supination as in lifting a weight. It occasionally locked in supination with a click. The patient was able to unlock the forearm by active pronation. These symptoms then gradually became less conspicuous, so that for the past nine years the wrist felt strong, comfortable and fully mobile and no longer locked. Clicking on rotation persisted.

On examination it was possible to produce a sudden anterior subluxation of the ulnar head accompanied by a clear click, at the mid-point of rotation. In the subluxated position there was anterior prominence of the ulnar head without radial deviation and a marked depression on the ulnar side of the posterior aspect of the wrist. On pronation reduction occurred spontaneously and smoothly at the mid-point of rotation. In the position of reduction there was marked antero-posterior mobility at the distal radio-ulnar joint, the excursion being about five-eighths of an inch (1.5 centimetres). The elbow, radio-ulnar and radio-carpal joints moved through the normal range. Radiographs confirmed the occurrence of subluxation and also showed an ununited fracture of the styloid process of the ulna. They showed no evidence of any other previous fracture of the radius or ulna. No treatment was advised.

\section{ANATOMICAL DISCUSSION}

The distal radio-ulnar joint for long has been described as if the ulnar head were immobile in rotation of the forearm (Johnston and Whillis 1949). Recently it has been emphasised that the axis of rotation of the forearm varies (Ray, Johnson and Jameson 1951; Burman 1953, 1956). If movement of the hand is resisted, the axial line will shift to become more congruent with the attempted axis of rotation of the hand, within the range of mobility of the two bones of the forearm. At one extreme-for example, with the small finger fixed in abduction-the axis will pass along a line taken through the centre of the radial head and the ulnar styloid process. This is the axis of rotation of the forearm that is usually described; but it is in fact one instance only-namely rotation of the radius on an all but unmoving ulna. As the line of transmitted force moves laterally the axis of rotation follows and may pass from the radial head to the radial styloid process so that the radius moves very little. In free rotation and in rotation with the hand in a normal comfortable working position, the axis lies more or less in a line projected from the radial head through the long or index fingers. Two components of the normal movement at the distal radio-ulnar joint in rotation of the forearm should therefore be distinguished. The ulnar head describes an arc in a coronal plane posterior to the distal end of the radius. The latter concurrently swings around the distal ulna through a wider arc. The greatest range of rotation of the forearm (with the elbow flexed to 90 degrees) is approximately 150 degrees (Salter and Darcus 1953). Any longitudinal movement or true rotation of the individual radius and ulna are conjectural; no true measurement of these has been recorded.

The normal checks to excessive rotation are not known for certain. Patrick (1946) considered that the final block to pronation was more proximal, at the crossed radial and ulnar shafts, but that the block to supination was the impingement of the postero-medial edge of the distal part of the radius against the extensor carpi ulnaris tendon in its groove. The function of the stoutest ligament - the triangular fibrocartilage attached to the base of the ulnar styloid and fanning out to the medial margin of the carpal articulating surface of the radius-is said to be to prevent diastasis (Gibson 1925, Milch 1926, Johnston and Whillis 1949). The relatively flimsy anterior and posterior distal radio-ulnar ligaments seem too weak to check rotatory or antero-posterior strains of any magnitude; and the medial [ulnar collateral] ligament is not aligned to control rotation.

The inferior radio-ulnar joint, accordingly, seems to be one with a considerable range of movement during rotation of the forearm, but to be provided with little to check any 
displacement except diastasis. Some features of simple antero-posterior displacement, shown in anterior dislocation, suggest that this description of the joint's function should be modified.

The dislocating force is an exaggeration of a normal force-transmitting movement of the limb, forced active supination on the fixed hand. Habitual dislocations occur similarly, in the course of active supination that most would consider light normal stress. The case of habitual subluxation described here is an extreme example since displacement occurred during

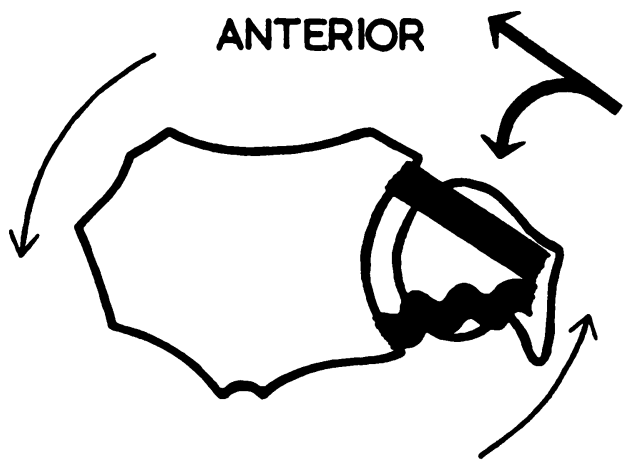

Fig. 5

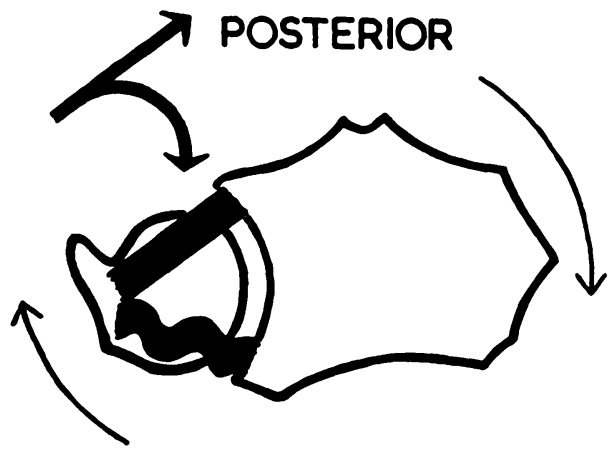

FiG. 6

End-on views of the right radio-ulnar joint in supination (Fig. 5) and in pronation (Fig. 6), showing the suggested function of the triangular fibrocartilage in rotation of the forearm. Lightly drawn arrows indicate rotation of the radius and ulna about the normal working axis of rotation of the forearm. Heavy arrows suggest the torque imparted to the ulnar head by the anterior and posterior bands of the triangular fibrocartilage, which tighten in phase with rotation.

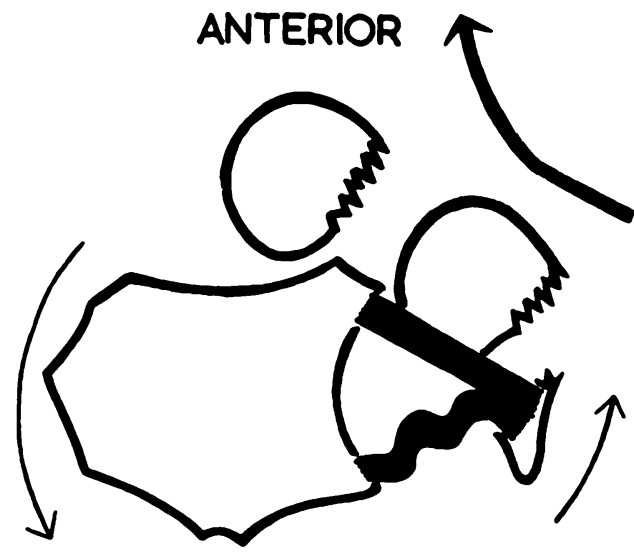

FIG. 7

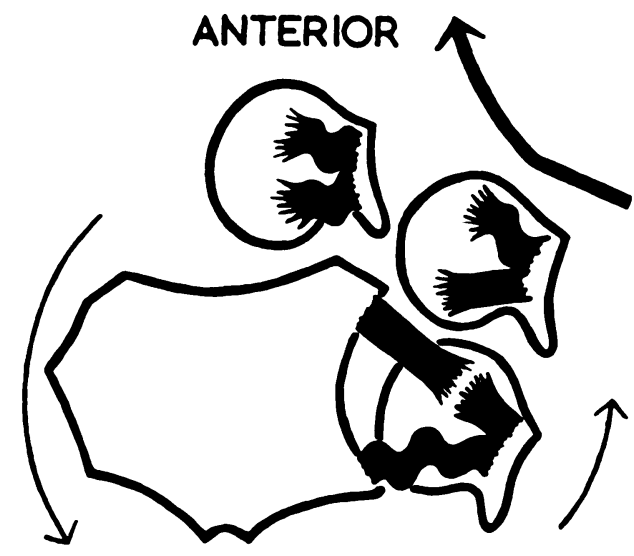

FIG. 8

End-on views of the right radio-ulnar joint in hypersupination, showing the suggested mechanism of anterior dislocation of the ulnar head with fracture of the ulnar styloid (Fig. 7), or rupture of the triangular fibrocartilage (Fig. 8).

simple free supination (Case 2). The commonplace, normal character of the stress, coupled with the rarity of the injury, suggests that a rupture or weakening of a strong check to displacement of the ulnar head occurs. Deformity of the radio-ulnar articular surfaces does not occur in these cases, as it does in the common complicated dislocations.

The role of the triangular fibrocartilage has to be considered. The attachment of this ligament to the base of the ulnar styloid process is not in the axis of normal working forearm rotation, but beyond it. It could only check antero-posterior displacement by a tangential pull on the ulnar head, tending to give this head an apparent rotation (relative to the distal end of the 
radius) into the more secure hollow of the ulnar notch. This contention is supported by the shape of the cartilage, which suggests that it is related to the two phases of forearm rotation rather than simply to some tendency to diastasis at the joint: it is attenuated in its middle sector and commonly fenestrated, with strong anterior and posterior margins. It has therefore the design of a mechanical couple. Thus in supination the anterior band would become taut, tending to rotate the ulnar head laterally and force the greater part of the head posteriorly. The reverse would occur in pronation (Figs. 5 and 6). Its rupture or laxity would allow anterior displacement in supination (Figs. 7 and 8). The ulnar styloid process is commonly fractured at its base in this dislocation, and when it is intact rupture of the fibrocartilage must be assumed. Most authors consider this to be a necessary component of the injury, although the opportunity to demonstrate it seldom occurs.

Two additional features seem to support this view. In extreme anterior dislocation the direction of displacement is always lateral. It is doubtful whether there is in fact in active rotation of the forearm any tendency to disatasis for the fibrocartilage to check. Secondly, the posterior and anterior distal radio-ulnar ligaments can be shown to become taut in phase with rotation-the posterior in pronation and the anterior at the extreme of supination (Davidson and Horwitz 1938). Their fibres are aligned with the posterior and anterior bands of the fibrocartilage, and it seems likely that they perform a similar function during rotation.

It is suggested therefore that the mechanical action of the fibrocartilage is not to check diastasis, but to maintain congruity of the radio-ulnar joint against the torsional stresses of rotation of the forearm. It is suggested that the fibrocartilage acts as a couple, imparting a torque to the ulnar head, and so holding it in the ulnar notch.

Mechanism of dislocation-When the hand is fixed, torsional stresses on the forearm act on the radius through the long lever of the hand and fingers. At the same time the axis of rotation of the forearm moves laterally away from the ulna. Such circumstances obtain only when the hand is fixed so that the radio-carpal joint's independence of the radio-ulnar joint is lost. When the force is great enough and if the hand remains fixed, the triangular fibrocartilage is torn or detached as supination forces the distal end of the radius off the ulna.

\section{SUMMARY}

1. Two cases are reported of the uncommon condition of simple anterior dislocation of the head of the ulna at the inferior radio-ulnar joint.

2. The literature concerning the condition and its treatment is reviewed.

3. The mechanism of the inferior radio-ulnar joint is discussed with particular reference to the function of the triangular fibrocartilage.

4. The mechanism of injury is discussed and a new idea of this mechanism is put forward.

I would like to thank Professor C. E. L. Allen for his advice and interest in this paper, Mr M. Singer for his helpful criticism, and $\mathrm{Mr}$ J. J. Commerell for permission to examine and describe the patient in Case 1, who was under his care.

\section{REFERENCES}

Burman, M. (1953): Primary Torsional Fracture of the Radius or Ulna. Journal of Bone and Joint Surgery, 35-A, 665.

Burman, M. (1956): Paralytic Supination Contracture of the Forearm. Journal of Bone and Joint Surgery, 38-A, 303.

Cotton, F. J., and Brickley, W. J. (1912): Luxation of the Ulna Forward at the Wrist (Without Fracture). Annals of Surgery, 55, 368.

Cox, F. J. (1942): Anterior Dislocation of the Distal Extremity of the Ulna. Report of a Case. Surgery, 12, 41.

CURR, J. F. and COE, W. A. (1946): Dislocation of the Inferior Radio-ulnar Joint. British Journal of Surgery, 34, 74.

Darrach, W. (1912): Forward Dislocation at the Inferior Radio-ulnar Joint. Annals of Surgery, 56, 801. 
Davidson, A. J., and Horwitz, M. T. (1938): Recurrent or Habitual Dislocation of the Inferior Radio-ulnar Articulation. American Journal of Surgery, N.S. 41, 115.

GiBson. A. (1925): Uncomplicated Dislocation of the Inferior Radio-ulnar Joint. Journal of Bone and Joint Surgery, 7, 180.

Goulden, R. P. (1954): Report on an Unusual Injury at the Wrist Joint. Journal of the Royal Army Medical Corps, $100,307$.

Hill, R. B. (1939): Habitual Dislocation of the Distal End of the Ulna. Report of a Case. Journal of Bone and Joint Surgery, 21, 780.

Johnston, T. B., and Whillis, J. (1949): Gray's Anatomy. Thirtieth edition, p. 479. London: Longmans, Green and Co.

Liebolt, F. L. (1950): A New Method for Repair of the Distal Radio-ulnar Ligaments. New York State Journal of Medicine, 50, 2,817.

MiLCH, H. (1926): Dislocation of the Inferior End of the Ulna. Suggestion for a New Operative Procedure. American Journal of Surgery, N.S. 1, 141.

Patrick, J. (1946): A Study of Supination and Pronation, with Especial Reference to the Treatment of Forearm Fractures. Journal of Bone and Joint Surgery, 28, 737.

Ray, R. D.. Johnson, R. J., and Jameson, R. M. (1951): Rotation of the Forearm. An Experimental Study of Pronation and Supination. Journal of Bone and Joint Surgery, 33-A, 993.

Regan, J. M., and BiCKel, W. H. (1945): Fascial Sling Operation for Instability of the Lower Radio-ulnar Joint. Proceedings of the Staff Meetings of the Mayo Clinic, 20, 202.

Salter, N., and Darcus, H. D. (1953): The Amplitude of Forearm and of Humeral Rotation. Journal of Anatomy, 87, 407.

Vol. 42 B, No. 3, AUGUST 1960 